\title{
An Optics Laboratory Module on Image Formation, Aberrations, and Lens Design
}

\author{
Robert D. Polak and Nicholas M. Pflederer
}

Department of Physics, Loyola University Chicago, Chicago, IL 60660

\begin{abstract}
To foster a deeper understanding of advanced laboratory concepts, weekly laboratories in an upper-division optics course have been replaced by modules, where students study a single topic over the course of several weeks. This culminates in a laboratory report written according to the American Institute of Physics style manual. One module covers image formation, aberrations and lens design. The students measure the mechanical properties of a lens and the optical properties of the glass. With this information, they build a virtual lens using ray-tracing software and compare results within the virtual environment to those measured in the laboratory.
\end{abstract}

Keywords: Educational Laboratory Experiments, Optical System Design, Aberrations, Ray-Tracing Software, Zemax PACS: 01.50.Pa, 42.15.Eq, 42.15.Fr

\section{INTRODUCTION}

Optics and Optics Laboratory are required upperdivision courses to complete the major in physics at Loyola University Chicago. In recent years, the laboratory course has been restructured with weekly laboratories on individual topics replaced by modules focused on a particular subtopic of optics. The laboratory work for each module now takes place over several weeks and culminates in a laboratory report written according to the American Institute of Physics style manual. ${ }^{1}$

This work describes the module on image formation, aberrations, and lens design. Students measure key mechanical properties of two lenses (one converging and one diverging) and key optical properties of the glass making up the landscape lens. These data are used as input into the ray-tracing software program Zemax. ${ }^{2}$ Image quality is studied as a function of key parameters such as wavelength, aperture size, image plane location and field angle; specific aberrations can also be identified. Aspects of these experiments performed in the virtual environment are then repeated in the laboratory. Students employ an achromatic doublet lens to greatly reduce chromatic aberrations, which allows for the study of coma and astigmatism both in the virtual laboratory and on the laboratory bench. Finally, the original converging lens is optimized in Zemax to minimize the effects of aberrations to satisfy particular specifications.

This module allows students to become familiar with the tools and techniques used to measure and study the mechanical and optical properties of the lens.
Students also learn how to use the ray-tracing software Zemax as a means to identify limitations of the lens, such as the presence of particular aberrations. Students compare results obtained with Zemax to those created in the physical laboratory and practice methods to reduce the aberrations.

\section{EXPERIMENTAL PROCEDURES}

\section{Measuring key properties of lens}

Students measure the key physical properties of one converging and one diverging lens. In our laboratory, students use one 3" diameter biconcave lens and one 3" diameter biconvex lens both made of N-BK7 glass with focal length of approximately $160 \mathrm{~mm}$. The radius of curvature of both sides of the lens is measured using a spherometer and a lens clock. The thickness and diameter of the lens is also measured using calipers.

To predict the optical performance of the lens, the optical properties of the glass must also be measured. Students use an Abbe refractometer ${ }^{3}$ and the method of minimum deviation ${ }^{4,5}$ to measure the index of refraction of a prism made of the same glass from which the lens is made. The source light is created using gas discharge tubes. To make it easier to detect the Fraunhofer $F$ spectral line using the Abbe Refractometer, the unwanted source light will be blocked using a bandpass filter. ${ }^{6}$ Using the measured values, students can correctly identify the glass type to be N-BK7. 

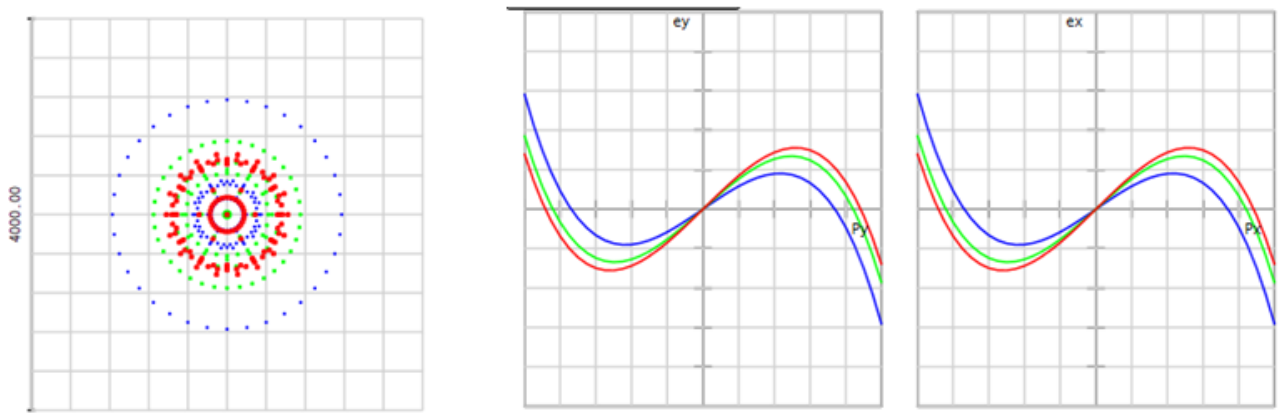

FIGURE 1. Images of the Zemax simulations of the spot diagram (left) and ray fan (right) for the converging lens for three wavelengths are shown above with a point source at infinity along the optical axis (field angle of $0^{\circ}$ ). In both images, red represents the hydrogen $\mathrm{C}$ spectral line, green represents the helium $\mathrm{d}$ spectral line and blue represents the hydrogen $\mathrm{F}$ spectral line. In the spot diagram, the horizontal and vertical axis represent distance with each individual block representing $400 \mu \mathrm{m}$. In the ray fan, the horizontal axis represents the normalized coordinate $\left(\mathrm{P}_{\mathrm{x}}\right.$ or $\left.\mathrm{P}_{\mathrm{y}}\right)$ which will range from -1 to 1 with the extreme representing the light passing at the edge of the entrance pupil and 0 represent the light passing through the center of the entrance pupil. The vertical axis represents the distance the light intersects the image plane relative to the location where the chief ray intersects the image plane, with each vertical block representing $400 \mu \mathrm{m}$. The left hand ray fan gives light traveling along the yaxis $\left(e_{\mathrm{y}}\right.$ vs. $\left.\mathrm{P}_{\mathrm{y}}\right)$ and the right hand ray fan gives light traveling along the $\mathrm{x}$-axis $\left(\mathrm{e}_{\mathrm{x}} \mathrm{vs} . \mathrm{P}_{\mathrm{x}}\right)$.

\section{Studying aberrations in Zemax}

The data collected are used as input into the Zemax ray-tracing software program to create a model of both the converging lens and diverging lens. When completed, the software will perform functions such as calculating the back and effective focal lengths. However, the primary educational benefit of using the software is to allow students to examine many optical phenomena quickly by creating light rays and mathematically calculating their path through the lens. Professional lens designers will use the spot diagram or ray $\operatorname{fan}^{7}$ generated by software to understand the performance of the lens and to identify key aberrations. ${ }^{8}$ An example of a spot diagram and ray fan are given in Fig. 1 for the lens based on data collected for the converging lens.

With the model in place, students can study the effects of changing key input parameters such as the location of the image plane, wavelength of incident light, entrance pupil diameter and the angle that light enters the lens from an object at infinity (called field angle). They will perform these studies using their virtual converging lens.
Initially, the students study the effects of wavelength of image formation. Because of dispersion, light from different wavelengths will focus at different points. Using wavelengths of light associated with the Hydrogen $\mathrm{F}$ and $\mathrm{C}$ spectral lines and Helium d spectral line and setting the field angle to $0^{\circ}$ and the entrance pupil diameter to $5 \mathrm{~mm}^{9}$, the students perform a 'quick focus' which places the image plane where the root mean squared value of the radial spot size is minimized, herein called the best focus. Figure 2 shows the spot diagrams of the three wavelengths closer to the lens than the best focus and farther from the lens from the quick focus. As expected, the $\mathrm{F}$ spectral line is focused closer to the lens than either the $\mathrm{d}$ or $\mathrm{C}$ spectral lines, representing an aspect of chromatic aberrations.

The students then study the effects of defocus and spherical aberrations. The software is set to use only the $\mathrm{d}$ spectral line, the field angle of $0^{\circ}$ and an entrance pupil diameter of $0.01 \mathrm{~mm}$, essentially eliminating all aberrations. Students measure the rms spot size as a function of distance of the image plane from the best focus. Next, the students return to the best focus and increase the diameter of the entrance pupil to the full

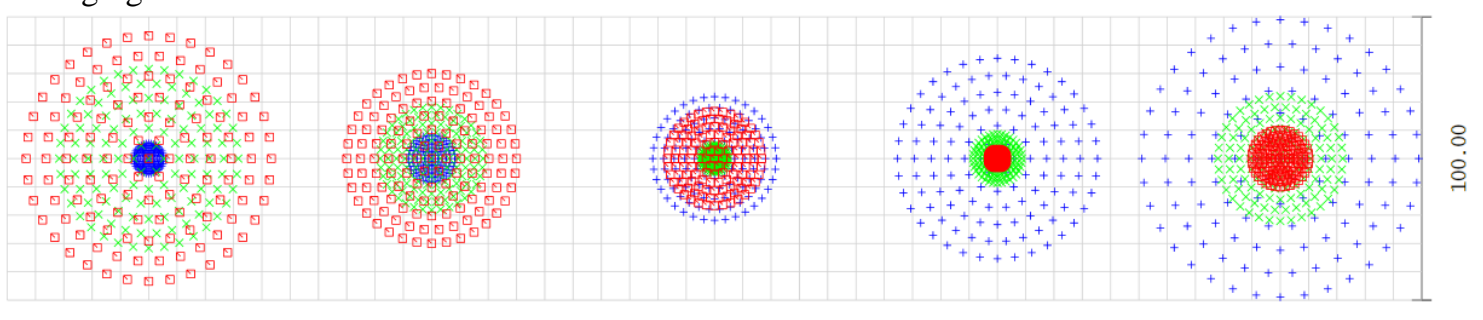

FIGURE 1. An image of the Zemax simulation is shown here. The five spot diagrams represent where light is incident upon different image planes with the middle diagram at the best focus. The two diagrams to the left of the best focus represent spot diagrams that are 1.0 (far left) and $0.5 \mathrm{~mm}$ closer to the lens than the best focus spot diagram. The two diagrams to the right of the best focus represent spot diagrams that are 1.0 (far right) and $0.5 \mathrm{~mm}$ farther from the lens than the best focus spot diagram. As seen here, the blue wavelength focuses first closest to the lens, the green then focuses further from the lens and finally the red focuses furthest from the lens. Note that the scale each division is $10 \mu \mathrm{m}$. 
diameter of the lens. Since the only Seidel monochromatic aberration present at a field angle of $0^{\circ}$ is the spherical aberration, the students observe how increasing the lens diameter increases this aberration.

\section{Laboratory Measurements of Converging and Diverging Lens}

Students return to the physical laboratory to test the results from the ray-tracing simulations. They will measure the focal lengths of both the converging and diverging lens using a lensmeter ${ }^{10}$ and the autocollimation method as described by Wyant. ${ }^{11}$ In our laboratory, the autocollimation method is performed using a uniform light source of light emerging from an open port of an integrating sphere ${ }^{12}$ with a 100 micron pinhole. For each lens, the students must observe the point where the light reflected formed a small spot at the same place where the pinhole is. As such, by tilting the mirror slightly, the reflected light forms a small visible dot next to the pinhole that can be observed directly.

Once they have measured the focal lengths of the two lenses, they will study aberrations using just the converging lens. A negative 1951 USAF test target is placed in front of the open point of the uniform light source. The object is placed along the optical axis of the converging lens and an image is formed on frosted glass behind the lens as shown in Fig. 3.

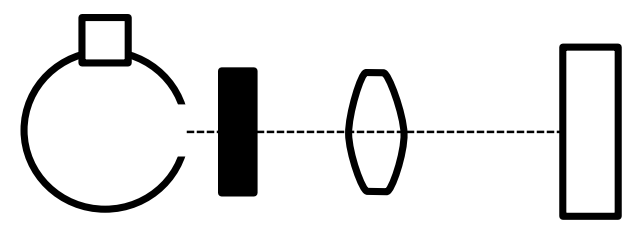

FIGURE 3. Light emerges from an open port of an integrating sphere (far left) passing through a pinhole or 1951 USAF test target and then the lens. An image is formed on frosted glass (far right).

There is a haze around the image formed as a result of spherical aberrations. An aperture placed in front of the lens acts as an entrance pupil. As the diameter of the aperture decreases, the haze decreases and the image becomes clearer. Typical results taken by a student using his cellular phone camera are shown in Fig. 4.

With the spherical aberrations reduced, students can then identify the presence of chromatic aberrations, which are noticeable along the clear lines of the image. A known solution to reducing chromatic aberrations is to use an achromatic doublet. The student will replace the converging lens with an achromatic doublet ${ }^{13}$ of similar focal length and note the disappearance of chromatic aberrations.
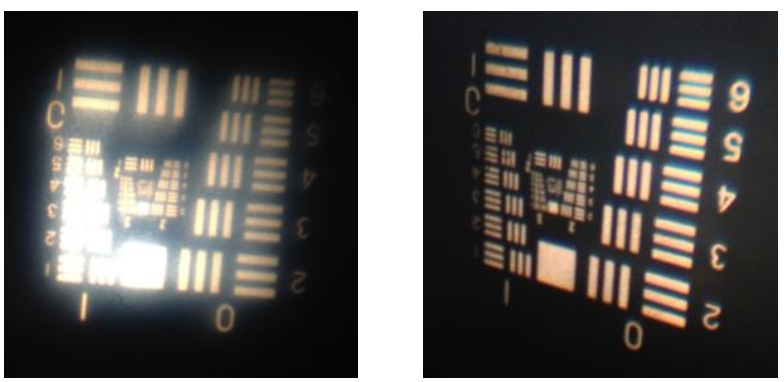

FIGURE 4. The image formed of the 1951 USAF test target through the converging lens without an aperture in place (left) and with an aperture reducing the entrance pupil (right). These images were taken with a student cellular phone camera.

\section{Coma and Astigmatism of the Doublet}

To observe other monochromatic aberrations, the students will use the achromatic doublet. The achromatic doublet exhibits high degrees of astigmatism or coma depending on which side is facing the source at non-zero field angles. Furthermore, ThorLabs provides Zemax files for their lens, so students can simulate the lens using Zemax as well as study the lens in the laboratory without having to repeat the mechanical and optical measurements from earlier in the module.

Using the set-up as shown in Fig. 3 with a 100 micron pinhole as the object, the object is placed 250 $\mathrm{mm}$ from the lens which has been placed on a motorized rotation stage. ${ }^{14}$ The image is formed on frosted glass. The same conditions are modeled within Zemax with the exception of the light being monochromatic (d spectral line). In both the laboratory and Zemax, the image is focused with a field angle at $0^{\circ}$ and the location of the image plane is not changed afterwards. Because we are using a pinhole as the object, the resulting image will closely match the spot diagram.

With the flatter side of the doublet towards the source, astigmatism is the dominant aberration. Figure 5 shows results in the laboratory and from software at a field angle of 15 degrees with a $25 \mathrm{~mm}$ aperture placed in front of the lens. The placement of the aperture is used to minimize the effects of other aberrations. 


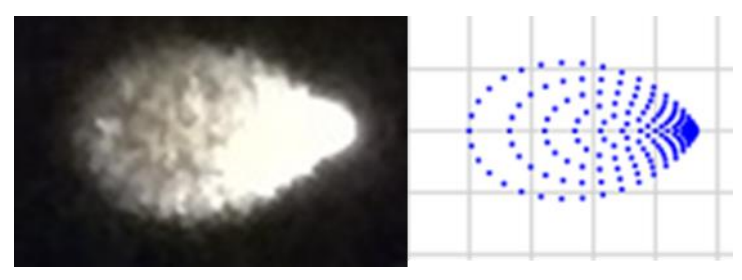

FIGURE 5. The image of a pinhole after passing through the doublet is shown on the right taken using a cell phone camera. The spot diagram simulated in Zemax is shown on the left. Astigmatism is present in both images. Each division on the spot diagram represents $2 \mathrm{~cm}$. The image taken by the camera was not measured.

With the more curved side of the doublet towards the source, coma is the dominant aberration. Figure 5 shows results in the laboratory and within the Zemax environment at a field angle of 5 degrees without an aperture placed in front of the lens.
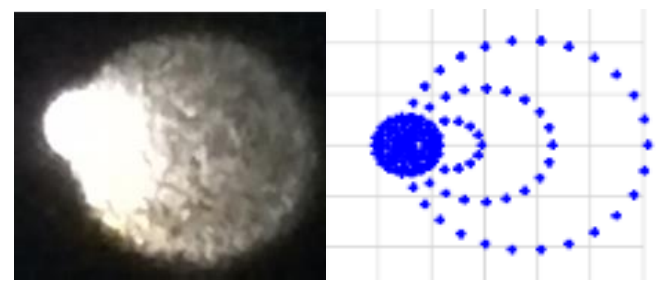

FIGURE 6. The image of a pinhole after passing through the doublet is shown on the right and the spot diagram simulated in Zemax is shown on the left. The coma aberration is present in both images. Each division on the spot diagram represents $6 \mathrm{~mm}$. The image taken by the camera was not measured.

\section{CONCLUSIONS}

This paper describes the module performed on image formation, aberrations and lens design as part of the Optics Laboratory course at Loyola University Chicago. This laboratory is completed over four weeks with minimal support from faculty members or laboratory assistants. After completion of the data collection, the students will write a laboratory report in the form of a journal paper using the American Institute of Physics style manual.

We have performed variations of this module for four years. The most experimental difficulty that students encounter is the proper use of the Abbe refractometer. Results on most aspects of the experiment are checked weekly by asking students to complete a writing assignment on the current week's experiment..

Those skilled in optics can find other opportunities to enhance and improve this laboratory module based on their own experience and the equipment they have available. There are also many examples ${ }^{15,}{ }^{16}$ on the internet of experiments where aberrations are studied in the laboratory and through the ray-tracing software.

\section{ACKNOWLEDGMENTS}

The authors would like to acknowledge the support of the Loyola University Chicago Department of Physics, especially Asim Gangopadhyaya and Fr. John Cunningham, S. J. that have supported this work. The authors would also like to acknowledge the Zemax Educational Support Program, which provides Zemax software for use in classes at reduced cost for educational purposes.

\section{REFERENCES}

1. AIP Style Manual, $4^{\text {th }}$ Ed., 1990.

2. Zemax Optical Studio 15 (Zemax, Kirkland, WA, 2015).

3. We use a Bellingham and Stanley Abbe 60 Abbe Refractometer.

4. J. R. Meyer-Arendt, Introduction to Classical and Modern Optics, $4^{\text {th }}$ Ed. (Prentice Hall, Upper Saddle River, NJ, 1995), pp.15-16.

5. We use a Cenco Enhanced Student Spectrometer for the measurement of the angle of minimum deviation.

6. We use a Newport 10BPF25-500 Bandpass Filter.

7. J. M. Geary, "Aberrations in General," in Introduction To Lens Design (Willmann-Bell, Inc., Richmond, 2011), pp. 65-67.

8. For an excellent introduction to aberrations and raytracing software, we suggest J. M. Geary, Introduction To Lens Design (Willmann-Bell, Inc., Richmond, 2011).

9. Reducing the entrance pupil makes it much clearer how the different wavelengths focus at different points when observing the spot diagrams.

10. We use a Reichert ML1 lensmeter.

11. J. C Wyant, "Measurement of Paraxial Properties of Optical Systems", http://fp.optics.arizona.edu/jcwyant/Optics513/ChapterN otes/Chapter01/Notes/Paraxial_Properties-Notes.pdf, date accessed July 2, 2015.

12. We use a 6" diameter general purpose integrating sphere with a 2" port (Labsphere 4P-GPS-053-SL) with an internal halogen light source (Labsphere IHLS-100).

13. We use a $150 \mathrm{~mm}$ focal length achromatic doublet from ThorLabs (AC508-150-A-ML).

14. We use a Newport URS100BPP motorized rotation stage with a Newport ESP301 motion controller.

15. E. E. Eyler, "Laboratory 3: Ray Tracing and Monochromatic Aberrations", http://www.phys.uconn.edu/ eyler/phys4150/N/P4150 la b3.pdf, date accessed July 2, 2015.

16. M. Nofzinger, "Lab 2: Aberrations" http://fp.optics.arizona.edu/nofziger/OPTI\%20202 L/OPTI202L\%20Lab2\%20Aberrations\%20SP12.pdf, date accessed July 2, 2015. 